\title{
La tutoría diversificada y flexible como estrategia institucional para la formación de los gerontólogos: el caso de la UAEH
}

\author{
Diversified and flexible tutoring as an institutional strategy for the training of Gerontology \\ students: the case of the UAEH \\ Lydia López Pontigo ${ }^{a}$, Bertha M. Pimentel Pérez, ${ }^{b}$, \\ Ma. del Refugio Acuña Gurrola ${ }^{c}$, Dulce A. Galindo Luna ${ }^{d}$
}

\begin{abstract}
:
Gerontology is a recent career that emerged in 2015 as an innovative proposal, which adds to the offer of the Autonomous University of the State of Hidalgo to train competent gerontologists in the integral intervention in the aging and old age process. For this, within the institutional training strategies the Tutoring Program arises as part of the actions of the institution itself, and its complemented by other programs depending on the specific group and individual characteristics and needs. Thus, this document is a theoretical review, as the first phase of action research which aims to describe sociodemographic factors that influence the school performance of gerontologists, in order to generate an intervention proposal that seeks to strengthen the academic training of these health professionals; through the proposal of a diversified and flexible tutoring, to influence the academic performance of the students of the gerontology degree comprehensively. In this way, the configuration of the object of study that concerns us takes as an axis of analysis the influence that sociodemographic factors have on the school performance of gerontologists, through a study with qualitative, descriptive methodology, through documentary analysis.
\end{abstract}

\section{Keywords:}

School performance, sociodemographic factors, tutoring

\section{Resumen:}

La Gerontología es una carrera reciente que surge en el 2015, como una propuesta innovadora, la cual se suma a la oferta de la Universidad Autónoma del Estado de Hidalgo para formar gerontólogos competentes en la intervención integral en el proceso de envejecimiento y la vejez. Para lo cual, dentro de las estrategias formativas institucionales se encuentra el Programa de Tutorías, como parte de las acciones de la propia institución, complementándose con otros programas en función de las características y necesidades específicas grupales o individuales. De esta manera, el presente documento es una revisión teórica, como primera fase de una investigación acción, el cual tiene como objetivo describir factores sociodemográficos que influyen en el rendimiento escolar de los gerontólogos, con la finalidad de generar una propuesta de intervención que busca fortalecer la formación académica de estos profesionales de la salud; a través de la propuesta de una tutoría diversificada y flexible, para incidir en el desempeño académico de los alumnos de la licenciatura de gerontología de manera integral. Así, la configuración del objeto de estudio que nos ocupa toma como eje de análisis la influencia que tiene

\footnotetext{
${ }^{a}$ Autor de Correspondencia, Universidad Autónoma del Estado de Hidalgo, Instituto de Ciencias de la Salud, https://orcid.org/0000-00016901-7909, Email: lydial@uaeh.edu.mx

b, Universidad Autónoma del Estado de Hidalgo, Instituto de Ciencias Básicas e Ingeniería, https://orcid.org/0000-0002-5266-6803, Email: bertha_pimentel@uaeh.edu.mx

Universidad Autónoma del Estado de Hidalgo, Instituto de Ciencias Básicas e Ingeniería, https://orcid.org/0000-0003-3194-5392, Email: maria_acuna@uaeh.edu.mx

d, Universidad Autónoma del Estado de Hidalgo, Instituto de Ciencias Básicas e Ingeniería, https://orcid.org/0000-0001-5012-8748, Email: dulce.galindo@uaeh.edu.mx
} 
el factor sociodemográfico en el rendimiento escolar de los gerontólogos, mediante un estudio con metodología cualitativa, descriptiva, a través de un análisis documental.

Palabras Clave:

Rendimiento escolar, factores sociodemográficos, tutoría

\section{Introducción}

La Universidad Autónoma del Estado de Hidalgo (UAEH) retoma la tutoría como estrategia institucional, ya que la educación superior, tanto a nivel nacional como mundial, enfrenta múltiples cambios que son generados por efectos estructurales en el plano económico, social, político y cultural que inciden en la lógica de funcionamiento del sistema educativo mexicano, el cual a su vez influye en la formación académica de los alumnos a causa de los procesos de enseñanza aprendizaje generados en las instituciones de educación superior específicamente en la UAEH [1] La educación superior es un bien de carácter estratégico, por el cual todos los Estados realizan grandes esfuerzos para ampliar su cobertura y diversificar su oferta pero, sobre todo, contar con una educación de calidad; en donde se busca que la principal característica de las instituciones sea aumentar la calidad de sus programas educativos, así como generar estrategias para incrementar el rendimiento escolar, eficiencia terminal y tasa de graduación. De esta manera, la Licenciatura en Gerontología que es una carrera relativamente joven, que en el 2015 emerge como una propuesta innovadora, se suma a la oferta de la $\mathrm{UAEH}$, formando gerontólogos competentes para conocer y aplicar los fundamentos teóricos, metodológicos y técnicos de las diversas vertientes de esta disciplina, de manera interdisciplinaria; a fin de resolver creativamente los problemas que enfrenta la población de la tercera edad en los diferentes espacios de intervención gerontológica.

Así, dentro de estrategias de formación institucionales de la UAEH se encuentra el Programa de Tutorías que, de manera específica, en la Licenciatura en Gerontología se vincula con otros programas.

De esta manera, este reporte, es la primera fase sobre el proyecto de investigación acción, que retoma el análisis documental descriptivo, con metodología cualitativa, de factores sociodemográficos que influyen en el rendimiento escolar los alumnos de la Licenciatura en Gerontología del Instituto de Ciencias de la Salud de la Universidad Autónoma del Estado de Hidalgo, que posibilita el planteamiento de una propuesta de intervención a través de la tutoría.
Por dicho motivo, esta investigación plantea el siguiente cuestionamiento:

- ¿Qué cualidades debe tener la tutoría como estrategia para disminuir el bajo rendimiento escolar de los alumnos de la Licenciatura de Gerontología?

Así, se emplea una metodología de tipo cualitativo, descriptiva, mediante el análisis de fuentes documentales formales e informales.

\section{Factores sociodemográficos de los alumnos de la Licenciatura en Gerontología de la UAEH}

Esta investigación en el marco de un Modelo Educativo constructivista y humanista, en donde el conocimiento no es adquirido sino desarrollado por los individuos, con base en sus características personales y contextuales, es decir, en función de factores sociodemográficos como lugar de residencia de origen y relaciones sociales informales, en especial las familiares.

En este sentido, la información aportada por los factores sociodemográficos permiten hacer un acercamiento a los estilos de vida de los alumnos, debido a que, a partir de éstos aparecen las primeras pistas para identificar posibles vulnerabilidades e, incluso, los factores de riesgo que deben ser tomados en cuenta en los planes y programas con la finalidad de impactar en el rendimiento escolar.

En el caso de los alumnos de la alumnos de la Licenciatura en Gerontología, las características contextuales de sus localidades de origen, como se muestran en la Figura 1, son heterogéneas; así, siete de cada diez alumnos de nuevo ingreso en 2017 la en el proviene de municipios diferentes a Pachuca y Mineral de la Reforma. Además, también se identifica la demanda por esta licenciatura no sólo entre los habitantes de Hidalgo, sino también de otros estados de las República como lo son Estado de México, Puebla, Tlaxcala, Guerrero, Veracruz, Querétaro y Zacatecas (Figura 


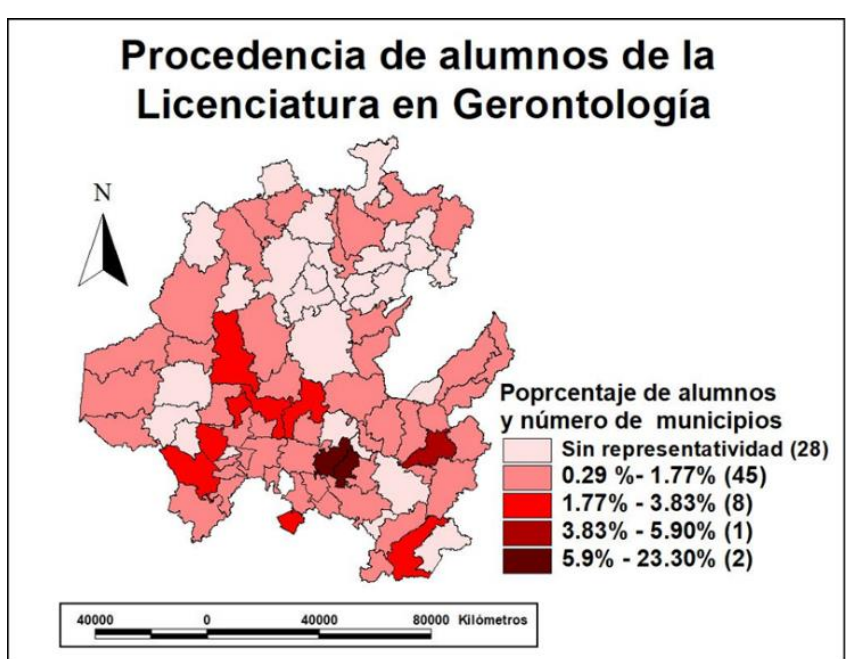

Figura 1. Municipios de procedencia de los alumnos de la Licenciatura en Gerontología.

Fuente: Elaboración propia a partir de la base de datos del Servicio Médico Universitario 2017.

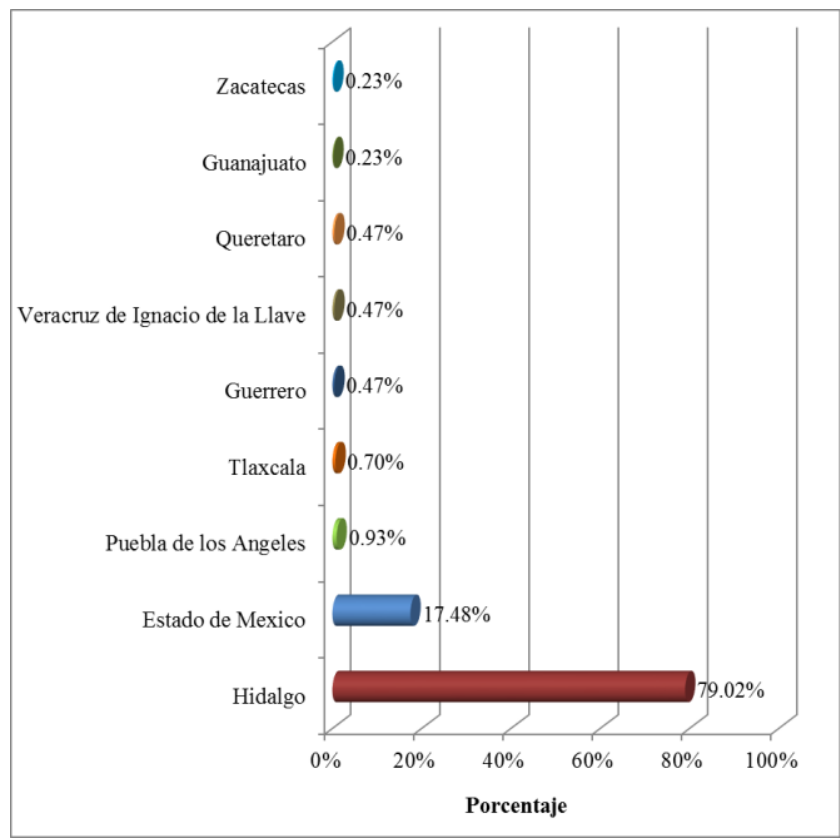

Figura 2. Estados de procedencia de los alumnos de la Licenciatura en Gerontología.

Fuente: Elaboración propia a partir de la base de datos del Servicio Médico Universitario 2017.

En este sentido, que los alumnos provengan de localidades geográficas diversas, implica una heterogeneidad de los elementos sociodemográficos, en los que se desarrollaron y vivieron antes de incorporarse a la vida universitaria, los cuales, de acuerdo con Carrión, son un factor predictor relevante para el rendimiento académico, ya que en esta variable se encuentran implícitos aspectos como el capital cultural, social y económico [2].
Otro elemento importante es el entorno inmediato en el que se encuentran los alumnos son las relaciones interpersonales familiares y no familiares, que pueden propiciar u obstaculizar resultados académicos positivos. De esta manera, en cuanto a la convivencia de los alumnos de la Licenciatura en Gerontología con diferentes miembros de red de apoyo social informal, como lo muestra la Figura 3, la relación más cercana es la que se establece con la mamá, es decir se hace presente una fuerte influencia del vínculo de protección; seguida de la relación con los hermanos, los cuales con frecuencia comparten características co-generacionales similares y, por tanto, de experiencias y referentes en cuanto prioridades sociales-culturales, los cuales no siempre son compatibles con el desarrollo académico y profesional. Así mismo, la relación con los amigos, aunque no tan cercana como con los hermanos, sin embargo, es destacable que la relación con los profesores es reportada en su mayoría como sólo buena e indiferente, por lo que, la falta de empatía e identificación con el profesorado puede ser un factor interviniente en la formación exitosa del gerontólogo.

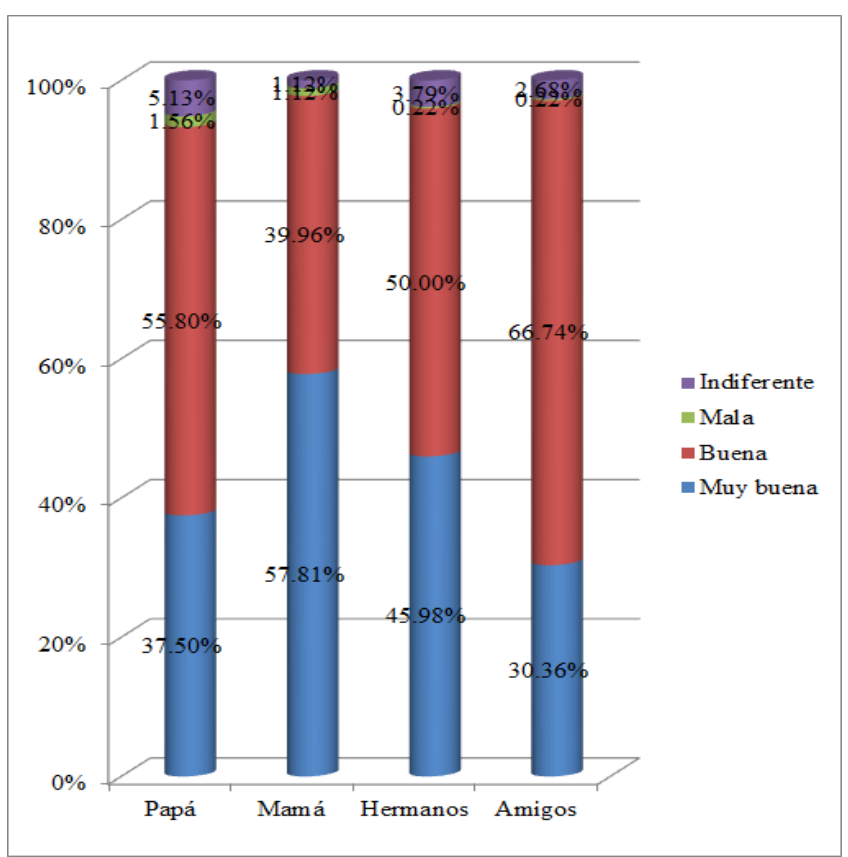

Figura 3. Relación de los alumnos de la Licenciatura en Gerontología con los miembros de su familia.

Fuente: Elaboración propia a partir de la base de datos del Servicio Médico Universitario 2017.

De esta manera, convergen e interaccionan los factores es cuando se vincula la procedencia de los alumnos y el grado de apego con la familia. Lo cual se debe, de acuerdo con Berger y Luckmann, a que la socialización primaria es la primera por la el individuo atraviesa y, por medio de ella, se convierte en miembro de la sociedad, 
razón por lo que es de mayor significancia para los sujetos [3]

\section{Rendimiento escolar en la Licenciatura en Gerontología de la UAEH.}

El rendimiento escolar es el grado de conocimientos que posee un alumnos, el cual se ve reflejado en la calificación, aprobatoria o desaprobatoria, en la asignaturas que cursa. [4,5]

Un mecanismo para dar seguimiento al estudiantado son las trayectorias escolares, dado que están vinculadas a la eficiencia terminal y, por ende, a los índices de aprobación/reprobación [6,7]

Índice de reprobación.

Que es el porcentaje de alumnos que no acreditan las asignaturas en evaluaciones ordinarias. Encontrando que la menor Tasa de Aprobación Ordinaria se presentó en primero, segundo y sexto semestre, pero todos con porcentajes superiores al $85 \%$, así como un $92.88 \%$ a nivel global (Figura 4).

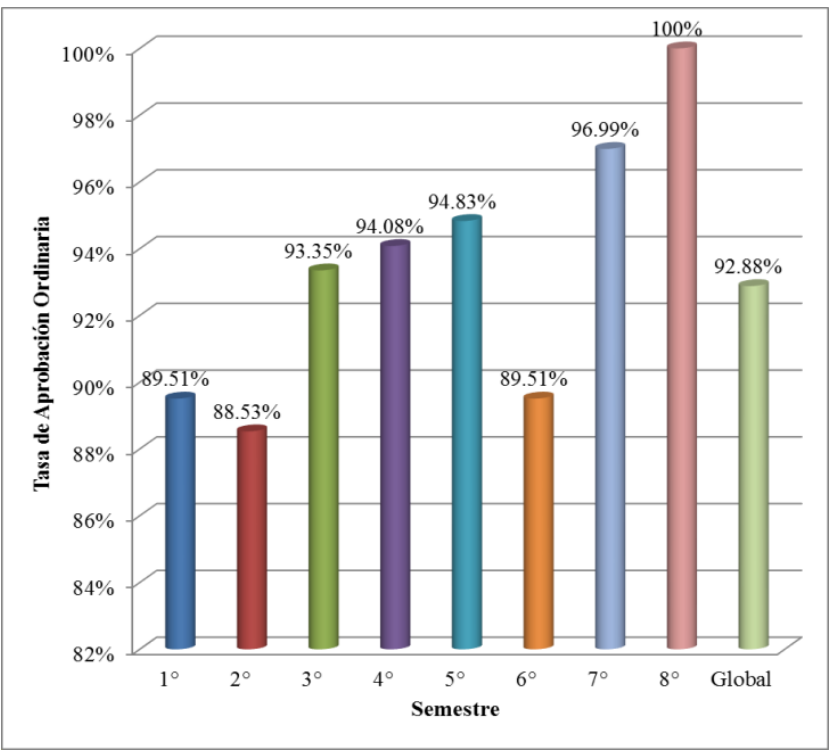

Figura 4. Tasa de Aprobación Ordinaria por semestre. Fuente: autoría propia.
Por otra parte, las asignaturas con una menor Tasa de Aprobación Ordinaria menor o igual al $80 \%$ fueron nueve, con una mayor frecuencia de aquellas relacionadas con la Lengua Extranjera, en diferentes semestres (Tabla 1).

Tabla 1. Asignaturas con una Tasa de Aprobación Ordinaria menor al $90 \%$.

\begin{tabular}{|c|c|c|}
\hline Semestre & Asignatura & $\begin{array}{c}\text { Tasa de } \\
\text { Aprobación }\end{array}$ \\
\hline \multirow[b]{3}{*}{$2^{\circ}$} & Eventos Pasados y & \\
\hline & $\begin{array}{c}\text { Futuros. Lengua } \\
\text { Extranjera }\end{array}$ & $72.97 \%$ \\
\hline & $\begin{array}{l}\text { Sujeto Gerontológico: } \\
\text { Una Lectura desde la } \\
\text { Filosofía, la Moral, la } \\
\text { Ética y la Bioética }\end{array}$ & $75.57 \%$ \\
\hline $3^{\circ}$ & $\begin{array}{c}\text { Logros y Experiencias. } \\
\text { Lengua Extranjera }\end{array}$ & $70.73 \%$ \\
\hline $5^{\circ}$ & $\begin{array}{c}\text { Causa y Efecto. Lengua } \\
\text { Extranjera }\end{array}$ & $77.27 \%$ \\
\hline \multirow{2}{*}{$6^{\circ}$} & $\begin{array}{l}\text { En Otras Palabras. } \\
\text { Lengua Extranjera }\end{array}$ & $62.50 \%$ \\
\hline & $\begin{array}{c}\text { Farmacología en el } \\
\text { Envejecimiento }\end{array}$ & $78.57 \%$ \\
\hline $7^{\circ}$ & $\begin{array}{c}\text { Optativa II (Desarrollo } \\
\text { Humano) }\end{array}$ & $54.55 \%$ \\
\hline
\end{tabular}

Fuente: autoría propia; con base en el seguimiento al de Trayectorias Escolares de los alumnos de la Licenciatura en Gerontología del periodo, enero-junio de 2018. 
Mientras que una de las herramientas para determinar la incidencia de las estrategias de enseñanza dentro del aula se evaluó la Tasa de Aprobación Ordinaria por grupo y asignatura (Tabla 2).

Tabla 2. Profesores con mayor Índice de Reprobación.

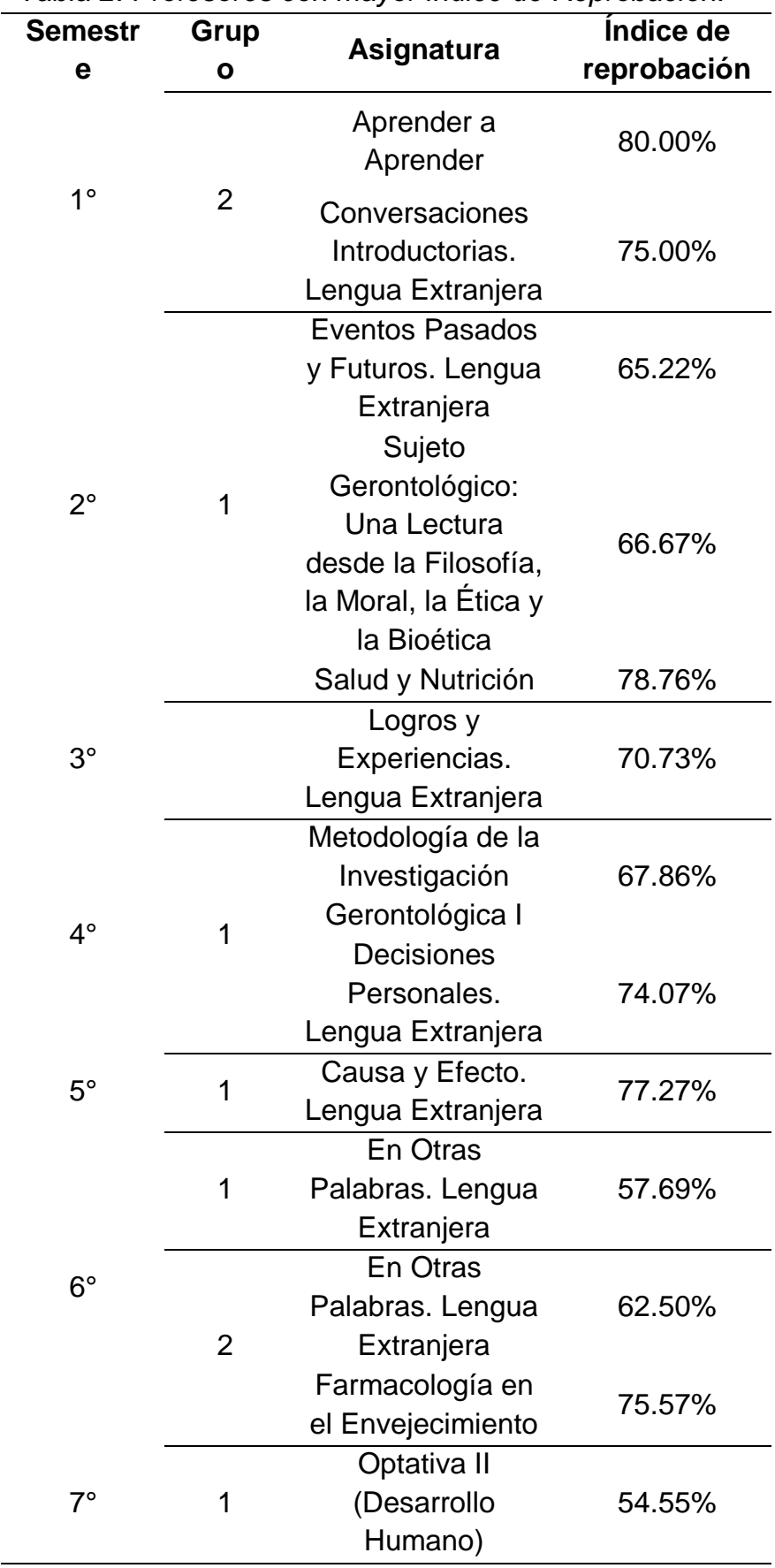

Fuente: autoría propia; con base en el seguimiento al de Trayectorias Escolares de los alumnos de la Licenciatura en Gerontología del periodo, enero-junio de 2018.

\section{Eficiencia terminal.}

De acuerdo con el Anuario estadístico de Población Escolar en la Educación Superior del ciclo escolar 2017 - 2018, de la Asociación Nacional de Universidades e Instituciones de Educación Superior (ANUIES), como se muestra en la Tabla 3, del ingreso nacional a los programas de estudio de licenciatura fue de 1,093,983 individuos con un total de egresados de 631,454 , lo que implicaría, que de ser una tasa de ingreso constante o relativa para cohortes anteriores, el porcentaje de egreso nacional aproximado sería de $57.72 \%$, y de 66.475 , si se parte del mismo supuesto a nivel estatal; proporción que se reduce en las disciplinas de salud nacional $(52.77 \%)$ y estatal $(50.99 \%)$ [7].

Tabla 3. Tasa de matrícula y egreso de programas de licenciatura.

\begin{tabular}{|c|c|c|c|c|}
\hline $\begin{array}{l}\text { Grado/discipli } \\
\text { na }\end{array}$ & Nivel & $\begin{array}{l}\text { Primer } \\
\text { Ingreso }\end{array}$ & $\begin{array}{l}\text { Matrícul } \\
a\end{array}$ & $\begin{array}{l}\text { Egresado } \\
s\end{array}$ \\
\hline \multirow[t]{2}{*}{ Licenciatura } & $\begin{array}{l}\text { Nacion } \\
\text { al }\end{array}$ & $\begin{array}{l}1,093,98 \\
2\end{array}$ & $\begin{array}{l}4,096,13 \\
9\end{array}$ & 631,454 \\
\hline & Estatal & 26,344 & 96,524 & 17,510 \\
\hline \multirow[t]{2}{*}{ Salud } & $\begin{array}{l}\text { Nacion } \\
\text { al }\end{array}$ & 118,237 & 456,370 & 62,396 \\
\hline & Estatal & 2,816 & 11,061 & 1,436 \\
\hline
\end{tabular}

Fuente: ANUIES. Anuario estadístico. Población Escolar en la Educación superior. Ciclo escolar 2017 - 2018.

En cuanto al Índice de Eficiencia Terminal de los alumnos de la Licenciatura en Gerontología de la UAEH, con base en datos obtenidos del Sistema de Información de Administración de Personal (SIAP) de la UAEH, del total de ingresados en cohorte o generación egresaron, en el tiempo estipulado, el $83.02 \%$, es decir por encima de los promedios nacionales de salud o de licenciatura en general. 8

Con base en lo expuesto, al analizar el rendimiento de los alumnos con base en la trayectoria escolar de los estudiantes de la educación superior, se hace necesaria una visión integral que contemple los factores que inciden en ella, como aspectos extraescolares, de manera específica los sociodemográficos, que pueden influir en el aprovechamiento e índice de aprobación 5-6

Los cuales deben ser contemplados en cualquier estrategia de formación, como lo es el programa de tutorías. 


\section{Propuesta de una tutoría diversificada y flexible.}

A pesar de que existen muchos conceptos diferentes de la tutoría, de manera general se retoma el término para definir a la acción orientadora, colectiva y coordinada, que el profesor tutor, junto con demás profesores y el propio centro educativo, proporciona a algún alumno o grupo de alumnos desde el actuar educativo. De acuerdo con dichos autores, la tutoría debe estar sujeta a una planificación y organización para que pueda ser una herramienta eficaz en la orientación de los estudiantes [8].

Ahora bien, el concepto de tutoría refleja la necesidad de que los programas educativos no se focalizan únicamente en actividades didácticas sino que debe proporcionar un acompañamiento al alumno en diferentes facetas, tanto académicas, sociales y personales. Debido a que, una vez que aún alumno inicia su educación universitaria, dichos elementos podrían convertirlo en un candidato de deserción escolar si no cuenta con el apoyo necesario al inicio, durante y al final de su proceso educativo [10].

Por esta razón, emanada de una gran tradición de apoyo al alumno universitario, la tutoría en la UAEH se ha ido transformado y adaptado a los nuevos requerimientos que demanda la sociedad del conocimiento, ante los grandes retos que enfrenta pues el perfil del profesional que se necesita formar actualmente es indispensable que sea integral, que potencialice las capacidades y fortalezca las debilidades de los educandos, con el fin de potencializar el aprovechamiento eficaz y adecuado de oportunidades para el rendimiento escolar de los alumnos; lo que implica para las universidades ya no sólo preocuparse por la formación disciplinar sino por la formación humanística y ética que se requiere, sobre todo en el área de la salud y específicamente en el área de gerontología. Ya que hoy, más que nunca, es necesario desarrollar el saber, el saber hacer, el ser y el saber convivir, para poder adaptarse a los escenarios cambiantes y globalizados que la sociedad presenta. En este contexto, y acorde a su Modelo Educativo es que la Universidad Autónoma del Estado de Hidalgo ha llevado a cabo la reestructura del Programa Institucional de Tutorías, a partir de un diagnóstico donde intervinieron tanto los agentes involucrados (tutores-tutorados) como los resultados de impacto del propio sistema, redefiniendo el objetivo general del mismo a fin de "contribuir en la formación integral del estudiante a través del acompañamiento de los docentes tutores, generando acciones y estrategias durante su trayectoria académica para coadyuvar a la permanencia y eficacia terminal", como lo indica el Programa Institucional de Tutorías (UAEH) [3].

En este diagnóstico se identificaron algunos factores que tienen fuerte incidencia en los índices de aprobación y permanencia y que coinciden con algunas de las principales razones personales, familiares y sociales de fracaso académico), las cuales son autoconocimiento, adaptación al medio universitario, motivación, autoestima, identidad universitaria y responsables del proceso. Por lo que, si son atendidos de manera temprana en el momento cuando ingresan los jóvenes al nivel universitario, su impacto puede disminuir e incluso en algunos casos desaparecer el efecto negativo. Siendo consistente con la visión constructivista y humanista que marca el Modelo Educativo de la UAEH y que es la base de la presente investigación, donde el centro de la tutoría es el estudiante, el Programa Institucional hace énfasis en tres momentos cruciales de la trayectoria escolar del educando: el ingreso, la permanencia y el egreso. Contando con los indicadores correspondientes para los alumnos de la Licenciatura en Gerontología [11].

\section{Índice de retención.}

Se calcula con base en los estudiantes de cada corte (generación) que de primer semestre se inscribe al segundo, empleando la fórmula de Guía para el Seguimiento de Trayectorias Escolares, de la Dirección General de Planeación de la UAEH. La cual se expresa de la siguiente manera con base en la fórmula de Guía para el Seguimiento de Trayectorias Escolares, de la Dirección General de Planeación de la UAEH. Así, en función de los datos obtenidos del Sistema de Información de Administración de Personal (SIAP) de la $\mathrm{UAEH}$, de un total 59 de la cohorte, 42 reingresaron a segundo semestre, es decir $71.19 \%$ [4].

Componentes de un Programa de Tutoría diversificada y flexible.

En cada uno de estos casos es necesario contar con tres tipos de tutoría, para dar un acompañamiento integral, considerando las tres esferas básicas en las que se desarrollan los estudiantes biológica, psicológica y social.

Los impactos en estas tres esferas (biológica, psicológica y social) ya los ha identificado la OMS (En red) como problemas prioritarios que aquejan a los jóvenes y siendo determinantes a nivel socioeconómico, cultural y emocional, donde podemos ubicar los factores 
extraescolares que inciden en el bajo rendimiento y deserción escolar de nuestros estudiantes son:

1. Aumento de adicciones en la población joven, con inicios en edades más tempranas.

2. Incremento de embarazos en la población de 15 a 19 años (madres solteras y abandono de estudios).

3. Incremento de la violencia y accidentes.

4. Trastornos de la alimentación.

5. Falta de actividad física.

6. Relaciones sexuales de riesgo [12].

Como se puede apreciar la complejidad de esta panorámica, aunada a los problemas de tipo educativo que los estudiantes presentan, inciden de manera importante en el comportamiento académico desarrollado a lo largo de su formación e incluyendo, el índice de reprobación, el desempeño escolar, y el rendimiento académico, entre otros. Por lo que, el desempeño académico de los alumnos universitarios se vuelve el centro de atención de la educación superior, dado el impacto positivo o negativo que representa no sólo para el joven que ve amenazado su futuro sino para la sociedad en general, al ser la educación el medio sine qua non o condición indispensable para lograr el desarrollo transgeneracional hacia una vida mejor [13].

En este sentido, autores como McKenzie y Schweitzar ubican una serie de factores relacionados con el desempeño académico de los estudiantes universitarios, los cuales se retoman en la presente investigación por considerar la vinculación que tienen con la problemática que se busca palear mediante la tutoría en el área académica de gerontología, con base en los cuatro factores que estos autores identifican: económicos, psicosociales, la apreciación cognitiva y demográficos. Cabe hacer mención que en este último se han agregado dentro de los componentes demográficos, el género y la etnicidad por el peso tan importante que dentro de nuestra cultural tienen (Tabla 4) [14].
Tabla 4. Factores relacionados con el desempeño académico de los estudiantes universitarios.

\begin{tabular}{|c|c|}
\hline Factores & Componentes \\
\hline Académicos & $\begin{array}{l}\text { Promedio del bachillerato, puntaje en el } \\
\text { examen de ingreso a la universidad, } \\
\text { número de intentos por abandonar una } \\
\text { carrera, promedio del primer año de } \\
\text { universidad }\end{array}$ \\
\hline Psicosociales & $\begin{array}{l}\text { Integración del estudiante al ambiente } \\
\text { universitario. Altos niveles de ansiedad, } \\
\text { redes de apoyo, pertenencia a algún } \\
\text { grupo de estudio, compromiso con sus } \\
\text { metas y con la institución. }\end{array}$ \\
\hline $\begin{array}{l}\text { Apreciación } \\
\text { cognitiva }\end{array}$ & $\begin{array}{l}\text { Percepción que tienen los estudiantes } \\
\text { de sus habilidades y desempeño }\end{array}$ \\
\hline & $\begin{array}{l}\text { académico. Percepción negativa, } \\
\text { percepción positiva, alta estima } \\
\text { académica, alta percepción de auto- } \\
\text { eficacia y bajos niveles de estrés. }\end{array}$ \\
\hline $\begin{array}{l}\text { Demográfico } \\
\text { s }\end{array}$ & $\begin{array}{l}\text { La edad, género, grupo étnico, } \\
\text { educación de los padres y asistencia } \\
\text { financiera. }\end{array}$ \\
\hline
\end{tabular}

Fuente: Tomado de: Octaviano García Robelo y Concepción Barrón Tirado. Un estudio sobre la trayectoria escolar... Perfiles Educativos, vol. XXXIII, núm. 131, 2011. IISUE-UNAM, p. 98 .[10].

De igual manera, se ha visualizado la importancia que tiene la propia institución formadora dentro de esta problemática, pues presenta una dualidad, tanto puede generar un ambiente para que el alumno culmine exitosamente sus estudios, como puede ser un factor de exclusión para motivar el abandono escolar. Como lo plantea Tinto (1987), dentro de las teorías de la deserción escolar organizacionales e interaccionales, aportan elementos para analizar los factores que pueden afectar la permanencia de los alumnos dentro de la institución, como lo son la infraestructura, la complejidad del diseño instruccional, los recursos económicos, el clima y los estímulos de la socialización presentes, pueden ser decisivos para su deserción. Es así, como la conducta estudiantil es vista como resultante de la interacción dinámica y recíproca amientes-individuos [15].

Todos estos factores no son lejanos a la situación por la que transitan los alumnos de gerontología donde predominan las mujeres ( $76 \%$ mujeres y $14 \%$ hombres). Estos jóvenes en menor o mayor medida enfrentan diversas situaciones que limitan su desarrollo académico, tal como lo demuestran los resultados del examen de ingreso aplicado por el CENEVAL, donde se puede encontrar promedios que van del 7.6 al $6.8 \mathrm{y}$ 
puntajes en las áreas de pensamiento matemático de 46, analítico de 44, estructura de la lengua de 50 y comprensión lectora de 49 según los índices del CENEVAL. Aunado a la incidencia de jóvenes que llegan con problemas de estrés, ansiedad, baja autoestima, depresión, o simplemente la carrera de gerontología no fue su primera opción. (Resultados EXANI II, enero-junio 2019 del Sistema de Administration de Programas Educativos, en red) $[10,11]$.

De esta manera, la complejidad de esta situación, ha generado bajo rendimiento escolar entre los estudiantes de gerontología, por ello se ha propuesto en el área académica llevar a cabo la integración de elementos que provienen de otros programas institucionales de la propia universidad al Modelo de Tutoría, que actualmente se aplica para atender de manera integral las necesidades de los estudiantes: Tutorías, Asesorías, Orientación Educativa y Atención Psicológica que incluye la Línea Garza para brindar un servicio de contención y escucha las 24 horas del día.

Así, el Programa de Tutorías en la UAEH está diseñado para dar respuesta a las necesidades particulares de los educandos, con un acampamiento integral en tres momentos con las siguientes modalidades:

Tutoría de adaptación al medio. Tiene como principal objetivo la retención de estudiantes de nuevo ingreso.

Tutoría de permanencia. Se centra en los estudiantes de segundo semestre en adelante, para apoyarle en el desarrollo de competencias para la vida.

Tutoría para el egreso. Como apoyo a los estudiantes se encuentran cerca de egresar de la licenciatura, quienes requieren de desarrollar habilidades y conocimientos que le permitan enfrentar la vida laboral.

Este tipo de tutorías se imparten de manera grupal e individual:

Grupales. Se le asigna a un profesor-tutor un grupo desde el inicio de semestre, así los jóvenes reciben de manera grupal la orientación necesaria para un buen desempeño académico e información sobre la normatividad, los servicios y los programas de apoyo que le pueden beneficiar.

Individuales. Se brinda atención personalizada al estudiante abordando situaciones personales, académicas y administrativas.

Por otro lado, un elemento innovador, sin duda, en el área de las TIC's es el Sistema Integral de Atención al Estudiante Universitario, que permite el registro de las tutorías y asesorías brindadas al educando a fin de dar seguimiento a cada grupo y caso que se atiende.

Ahora bien, las acciones que se llevan a cabo por parte del Programa de Tutorías necesitan articularse con otros programas igualmente importantes, pero que se ejecutan con base en sus propios objetivos. Por lo que es necesario llevar a cabo la concatenación de todas estas acciones, a partir de redefinir la concepción que se tiene de la tutoría, para hacerla diversificada en tanto los múltiples programas que deben integrarse y flexible considerando las diferentes instancias académicoadministrativas que se involucran, así como el cambio de roles que los actores involucrados.

El reto no es menor, pero se tienen que asumir dado que el costo es mayor si se continúa con los mismos modelos y sistemas de tutorías, que hasta ahora se han aplicado. Depositar la tutoría en un sólo actor rebaza la capacidad del mismo, se tiene que pensar en ella como una función sistémica que involucra no tanto a las instancias educativas como a la familia y la sociedad en general. Razón por lo cual esta investigación pretende aportar herramientas para coadyuvar en la elucidación de la compleja realidad bio-psico-social de los alumnos de gerontología, mediante la presentación del siguiente modelo (Figura 5).

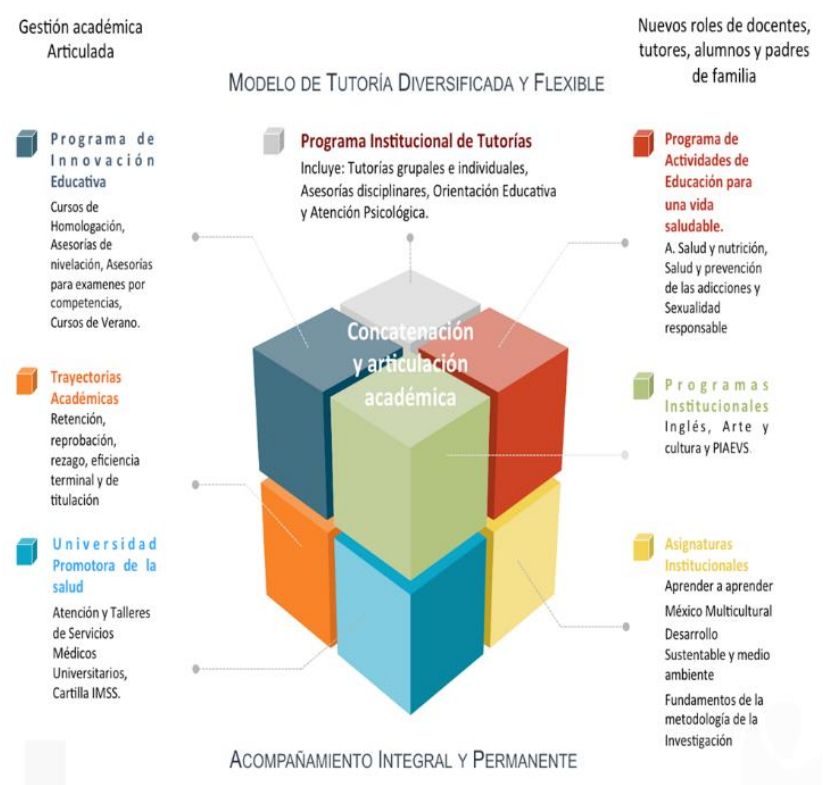

Figura 5. Modelo de tutoría diversificada y flexible. Fuente: autoría propia. 


\section{Referencias}

[1] Universidad Autónoma del Estado de Hidalgo. [homepage en Internet]. Pachuca: Programa Institucional de Tutorías; c2018 [citado 12 de agosto 2019]. Disponible en https://www.uaeh.edu.mx/division_academica/innovacionacademica/docs/programas-asignaturas/asesoria-ytutorias/programa-asesoria-academica-2018.pdf.

[2] Carrión E. Validación de características al ingreso como predictores de rendimiento académico en la carrera de medicina, Revista Cubana de Educación Médica Superior [Revista on-line] 2002; 1(16): [aprox. 2 p.]. Disponible en http://scielo.sld.cu/scielo.php?pid=S0864-

21412002000100001\&script=sci_arttext

[3] Berger P, Luckmann, T. La construcción social de la realidad. Buenos Aires: Amorrortu editores; 2003.

[4] Chain R, Ramírez M. Trayectoria Escolar: la Eficiencia Terminal en la Universidad Veracruzana en Revista de la Educación Superior, ANUIES [Revista on-line] 1997; 102(26): [aprox. 10 p.]. Disponible http://publicaciones.anuies.mx/pdfs/revista/Revista102_S2A2E S.pdf.

[5] Gutiérrez-García AG, Granados-Ramos DE, Landeros-Velázquez MG. Indicadores de la trayectoria escolar de los de psicología de la Universidad Veracruzana, Actualidades Investigativas en Educación [Revista on-line] 2011; 11(3): [aprox. 30 p.]. Disponible https://www.researchgate.net/publication/237034877_INDICA DORES_DE_LA_TRAYECTORIA_ESCOLAR_DE_LOS_A LUMNOS_DE_PSICOLOGIA_DE_LA_UNIVERSIDAD_VE RACRUZANA.

[6] Benítez MG, Becerra G, Soto RM, Aguilar MT. Trayectorias escolares de los estudiantes de la generación 1998 y 1999 de la Facultad de Contaduría y Administración de la UASLP, Revista de la Educación Superior [Revista on-line] 2004; XXXIII(131) [aprox. $47 \quad$ p.]. Disponible en: http://publicaciones.anuies.mx/pdfs/revista/Revista131_S1A1E S.pdf

[7] Asociación Nacional de Universidades e Instituciones de Educación Superior (ANUIES). [homepage en Internet]. México: ANUIES; c2018 [citado 09 de agosto 2019]. Disponible en http://www.anuies.mx/iinformacion-y-servicios/informacionestadistica-de-educacion-superior/anuario-estadistico-deeducacion-superior

[8] Universidad Autónoma del Estado de Hidalgo. Dirección General de Planeación [homepage en Internet]. Pachuca: Guía de Trayectorias Escolares; c2013 [citado 12 de agosto 2019]. Disponible

http://intranet.uaeh.edu.mx/DGP/pdf/2_guia_trayectoria.pdf.

[9] Universidad Autónoma del Estado de Hidalgo. [homepage en Internet]. Pachuca: Sistema de Información de Administración de Personal; c2018 [citado 12 de agosto 2019]. Disponible en http://sistemas.uaeh.edu.mx/dap/cons_empleados/index.php.

[10] García O, Barrón C. Concepción Barrón Tirado. Un estudio sobre la trayectoria escolar, Perfiles Educativos [Revista on-line]. 2011 XXXIII(131): [aprox. 19 p.]. Disponible en: https://www.redalyc.org/pdf/132/13218531007.pdf

[11] Universidad Autónoma del Estado de Hidalgo. [homepage en Internet]. Pachuca: Sistema de Administración de Programas Educativos, Resultados de EXANI II, enero-junio 2019; c2018 [citado 12 de agosto 2019]. Disponible en http://sistemas3.uaeh.edu.mx/sape/cenevalDependencia/index.p hp.

[12] Organización de las Naciones Unidas para la Educación, la Ciencia y la Cultura, UNESCO. [homepage en Internet]. México: Conferencia mundial sobre la educación superior; c1998 [citado 13 de agosto 2019]. Disponible en: http://www.unesco.org/education/educprog/wche/declaration_s pa.htm.

[13] Barranco SM, Santacruz MC. Los egresados de la UAA: trayectoria escolar y desempeño laboral [monografía en Internet]. México: 1995.

[14] McKenzie M, Schweitzar R. Who Succeeds at University? Factors predicting academic performance in first year Australian university students, Higher Education Research [Revista online]. 2001; 20(1): [aprox. 12 p.]. DOI: $10.1080 / 07924360120043621$

[15] Tinto V. Reflexiones sobre el abandono de los estudios superiores, Perfiles Educativos [Revista on-line] 1993; 3(7): [aprox. 17 p.]. Disponible en: https://biblat.unam.mx/es/revista/perfileseducativos/articulo/reflexiones-sobre-el-abandono-de-losestudios-superiores 